\title{
New Parameter of the Second Half of the P-Wave, P-Wave Duration, and Atrial Conduction Times Predict Atrial Fibrillation during Electrophysiological Studies
}

\author{
Raimundo Carmona Puerta ${ }^{a}$ Elizabeth Lorenzo Martínez ${ }^{\text {b }}$ \\ Magda Alina Rabassa López-Callejac Gustavo Padrón Peña ${ }^{c}$ \\ Yaniel Castro Torres ${ }^{d}$ Juan Miguel Cruz Elizundia ${ }^{a}$ \\ Fernando Rodríguez González ${ }^{a}$ Luis Ángel García Vázquez ${ }^{e}$ \\ Elibet Chávez González ${ }^{a}$
}

\begin{abstract}
aDepartment of Electrophysiology and Arrhythmology, Cardiovascular Hospital "Ernesto Guevara", Santa Clara, Cuba; ' Department of Physiology, Medical University of Villa Clara, Santa Clara, Cuba; ' Chief Professor in Cardiology, Cardiovascular Hospital "Ernesto Guevara", Santa Clara, Cuba; 'Coronary Care Unit, San Juan de Dios Hospital, Santiago de Chile, Santiago, Chile; 'Study Center of Physical Culture and Sport, Central University of Las Villas, Santa Clara, Cuba
\end{abstract}

\section{Highlights of the Study}

- Atrial fibrillation that occurs during electrophysiological studies is an indicator of electrical vulnerability.

- We report a new predictor of atrial fibrillation within the second half of the P-wave.

- The new parameter, maximum Ppeak-Pend, is an independent predictor of atrial fibrillation in electrophysiological studies.

- Atrial conduction times predict the occurrence of atrial fibrillation during electrophysiological studies better than $\mathrm{P}$-wave parameters.

\section{Keywords}

Atrial conduction time $\cdot$ P-wave $\cdot$ Electrocardiogram · Atrial fibrillation - Electrophysiological study

\section{Abstract \\ Objective: Several P-wave parameters reflect atrial conduc- tion characteristics and have been used to predict atrial fi- brillation (AF). The aim of this study was to determine the relationship between maximum $\mathrm{P}$-wave duration (PMax)}

and new P-wave parameters, with atrial conduction times (CT), and to assess their predictive value of AF during electrophysiological studies (AF-EPS). Subjects and Methods: This was a cross-sectional study in 153 randomly selected patients aged 18-70 years, undergoing EPS. The patients were divided into 2 groups designated as no AF-EPS and AFEPS, depending on whether AF occurred during EPS or not. Different P-wave parameters and atrial CT were compared for both study groups. Subsequently, the predictive value of the P-wave parameters and the atrial CT for AF-EPS was eval- karger@karger.com www.karger.com/mpp

Karger $\stackrel{\text { ' }}{5}$ GOPEN ACCESS
(C) 2021 The Author(s)

Published by S. Karger AG, Basel

This is an Open Access article licensed under the Creative Common Attribution-NonCommercial-4.0 International License (CC BY-NC) (http://www.karger.com/Services/OpenAccessLicense), applicable to the online version of the article only. Usage and distribution for commercial purposes requires written permission.
Correspondence to:

Raimundo Carmona Puerta, endotelio1975@gmail.com 
uated. Results: The values of CT, PMax, and maximum PpeakPend interval (Pp-eMax) were significantly higher in patients with AF-EPS. Almost all P-wave parameters were correlated with the left CT. PMax, Pp-eMax, and CT were univariate and multivariate predictors of AF-EPS. The largest ROC area was presented by interatrial CT $(0.852 ; p<0.001$; cutoff value: $\geq 82.5 \mathrm{~ms}$; sensitivity: $91.1 \%$; specificity: $81.1 \%$ ). Pp-eMax showed greater sensitivity (79.5\%) to discriminate AF-EPS than PMax $(72.7 \%)$, but the latter had better specificity (60.4\% vs. $41.5 \%)$. Conclusions: Left atrial CT were directly and significantly correlated with PMax and almost all the parameters of the second half of the P-wave. CT, PMax, and PpeMax (new parameter) were good predictors of AF-EPS, although CT did more robustly.

(C) 2021 The Author(s).

Published by S. Karger AG, Basel

\section{Introduction}

Various parameters derived from the $\mathrm{P}$-wave have been reported to predict atrial fibrillation $(\mathrm{AF})[1-3]$ and are therefore considered as "intermediate phenotypes" of this arrhythmia [4]. Most researchers agree that these parameters reflect atrial conduction characteristics, one of the most representative being the P-wave duration [5]. Some of these parameters have shown a direct correlation with intra-atrial and interatrial conduction time or other electrophysiological parameters reflecting conduction disturbances [6-11].

Only a few studies have used electrophysiological studies (EPS) to evaluate the degree of involvement of atrial conduction in the genesis of the different $\mathrm{P}$-wave parameters [6]. It would be useful to know the ability to predict $\mathrm{AF}$ based on different $\mathrm{P}$-wave parameters in the same study where direct measurements of atrial conduction are available. This would be particularly valuable if this arrhythmia has not been clinically expressed and there is only a state of increased electrical vulnerability. The aim of this study was to determine the relationship between the maximum $\mathrm{P}$-wave duration and new parameters of the second half of the P-wave, with atrial conduction time, and to evaluate their value as predictors of $\mathrm{AF}$ during EPS (AF-EPS).

\section{Subjects and Methods}

This was a cross-sectional study that included 153 patients aged 18-70 years. These patients were randomly selected from a study population consisting of 286 patients with a clinical history of palpitations and a confirmed diagnosis of atrioventricular node re-entry tachycar- dia (AVNRT) or accessory pathways, who underwent EPS and endocardial mapping in the Cardiac Electrophysiology Lab of the Cardiovascular Hospital "Ernesto Guevara," Santa Clara, Cuba, between June 2017 and February 2020. Only patients without significant cardiac anomalies and/or a previous history of AF were included.

The patients were divided into 2 groups designated as AF-EPS and no AF-EPS, defined as patients who had AF and patients who had no AF during EPS, respectively (for definition of "AF during EPS," see below). Different P-wave parameters derived from electrocardiogram and atrial conduction times were compared in both study groups. Subsequently, the correlation between P-wave parameters or atrial conduction times and AF-EPS was evaluated.

The EPS was performed after at least $6-8 \mathrm{~h}$ of fasting and without antiarrhythmic medication, at least for 5 or more half-lives of the drug. All subjects were assessed by transthoracic echocardiography for exclusion of cardiac anomaly.

\section{Exclusion Criteria}

(1) More than 2 electrocardiographic leads that did not allow measurement of the $\mathrm{P}$-wave and/or any channel of the atrial intracavity records with poor signal quality. (2) Permanent ventricular pre-excitation (excluded because of the difficulty of measuring the P-wave offset).

\section{Study Variables}

The following general variables were taken into account: age, gender, arterial hypertension, diabetes mellitus, and the occurrence of AF-EPS. AF-EPS was considered to be present if paroxysms lasting $>10$ s (induced spontaneously or by catheter manipulation) were documented. Stimulation techniques were not used for its induction. In this study, the occurrence of AF-EPS was used as an indicator of electrical atrial vulnerability.

\section{P-Wave Parameters Studied}

Maximum $\mathrm{P}$-wave duration (PMax) is the longest $\mathrm{P}$-wave duration in any of the 12 leads of the electrocardiogram. Maximum Ppeak-Pend interval (Pp-eMax) is the greatest value of the time between the peak of the P-wave and its end, in any of the 12 leads of the electrocardiogram (new P-wave parameter). Ppeak-Pend dispersion (Pp-eDis) was determined by subtracting the lowest Ppeak-Pend interval from Pp-eMax, taking into account the 12 leads of the electrocardiogram (new P-wave parameter).

Up to 3 consecutive P-waves were measured and averaged in each of the 12 leads. Electrocardiographic records were obtained at a calibration of $20 \mathrm{~mm} / \mathrm{mV}$ and a sweep speed of $50 \mathrm{~mm} / \mathrm{s}$. The onset of the P-wave is defined as the point of first detectable upward or downward slope from the isoelectric line for positive or negative waveforms, respectively. Return to the isoelectric line is considered as the end of the P-wave. If the start or end of the Pwave was not clearly defined, that lead was excluded. All electrocardiographic measurements were expressed in milliseconds. In leads with positive $\mathrm{P}$-wave and without notches, the most prominent elevation was considered as its peak (in case of notch, its deepest point was chosen as the equivalent of the peak), and in case of negative $\mathrm{P}$-wave, the nadir was taken. In the case of $\mathrm{P}$-wave \pm as in $\mathrm{V} 1$, the negative terminal portion was taken as its second half.

\section{Electrophysiological Parameters Studied}

P-proximal coronary sinus interval (P-PCS) was measured from the onset of the P-wave to the onset of the earliest reproduc- 
Table 1. Baseline characteristics of all patients and stratified by arrhythmic substrates

\begin{tabular}{lcccc}
\hline & $\begin{array}{l}\text { All patients } \\
(N=153)\end{array}$ & $\begin{array}{l}\text { AVNRT } \\
(N=83)\end{array}$ & $\begin{array}{l}\text { Accessory pathways } \\
(N=70)\end{array}$ & $p$ value \\
\hline Age, years & $39.53 \pm 14.36$ & $43.40 \pm 13.83$ & $34.94 \pm 13.70$ & $0.001^{*}$ \\
Male sex & $58(37.91)$ & $22(26.51)$ & $36(51.43)$ & $0.002^{*}$ \\
AF-EPS & $45(29.41)$ & $17(20.48)$ & $28(40.00)$ & $0.026^{*}$ \\
Hypertension & $36(23.53)$ & $27(32.53)$ & $9(12.86)$ & 1.000 \\
Diabetes mellitus & $4(2.61)$ & $3(3.61)$ & $1(1.43)$ & 0.146 \\
Body weight, kg & $69.39 \pm 10.01$ & $68.49 \pm 9.62$ & $70.50 \pm 10.44$ & 0.146 \\
Heart rate, bpm & $82.05 \pm 20.50$ & $82.55 \pm 21.80$ & $81.41 \pm 18.86$ & 0.737 \\
Echocardiographic findings, mm $\quad$ & $33.65 \pm 4.56$ & $33.86 \pm 4.36$ & $33.39 \pm 4.82$ & 0.522 \\
$\quad$ LA size & $27.76 \pm 3.81$ & $28.01 \pm 3.78$ & $27.45 \pm 3.85$ & 0.365 \\
\hline RA size & &
\end{tabular}

AF, atrial fibrillation; AF-EPS, atrial fibrillation in the electrophysiological study; AVNRT, atrioventricular nodal re-entrant tachycardia; LA, left atrium; RA, right atrium. * Only significant variables were included in the analysis.

ible rapid deflection of the atrial electrogram recorded from the proximal poles of a decapolar catheter (BIOTRONIK) positioned into the coronary sinus (most proximal record within the area of the coronary sinus ostium). The exact position of the coronary sinus ostium was defined using its relationship with the HIS catheter (defining the septum) and the presence of typical coronary sinus records (interelectrode distance of the same pair, $5 \mathrm{~mm}$; distance between pairs, $10 \mathrm{~mm}$ ). This parameter was accepted as a measure of the intraright atrial conduction time from the sinus node to the respective recording area.

P-distal coronary sinus interval (P-DCS) was measured from the onset of the $\mathrm{P}$-wave to the onset of earliest reproducible rapid deflection of the atrial electrogram recorded from the distal poles of a decapolar catheter (BIOTRONIK) positioned into the coronary sinus around the left lateral portion of the mitral ring (interelectrode distance of the same pair, $5 \mathrm{~mm}$; distance between pairs, $10 \mathrm{~mm}$ ). This parameter was accepted as a measure of interatrial conduction time.

Left intra-atrial conduction time (DCS-PCS) was calculated as the difference of P-DCS - P-PCS. The measurements were performed in sinus rhythm by an experienced electrophysiologist, blinded to the data of each case and with the patient awake, without the effect of anesthetics or isoprenaline, and before the application of radiofrequency, on an EP TRACER multichannel polygraph (CardioTek, Maastricht, The Netherlands). Electrophysiological measurements were made manually using electronic calipers at a sweep speed of $300 \mathrm{~mm} / \mathrm{s}$ and expressed in milliseconds. The catheter positions were monitored using biplane fluoroscopy with standard right anterior oblique and left anterior oblique views.

The echocardiographic parameters studied included left atrium size (anteroposterior) and right atrium size (minor axis), measured according to current recommendations from the American Society of Echocardiography and the European Association of Cardiovascular Imaging [12]. The echocardiographic study was performed prior to the EPS according to the protocol established in the institution.

\section{Statistical Analyses}

For all analyses, commercially available computer software (SPSS Version 21.0, SPSS Inc., Chicago, IL, USA) was used. For the comparison of quantitative variables with normal distribution, the Student's $t$ test was used, and for quantitative variables with nonparametric distribution, the Mann-Whitney U test was used. The normality test was performed for continuous data using a Kolmogorov-Smirnov test with Lilliefors correction. The comparison of categorical variables was carried out using the $\chi^{2}$ test. Correlations were performed by estimating Pearson's correlation coefficient, while binary logistic regression was used for univariate and multivariate analysis. The determination of sensitivity and specificity of the predictors derived from the logistic regression analysis was carried out by calculating receiver operating characteristic (ROC) curves. The cutoff points were determined by calculating the Youden index. Values of $p<0.05$ were set as the minimum level of statistical significance throughout the study.

\section{Results}

\section{Baseline Characteristics}

Of the 153 cases studied, the majority presented AVNRT and less than half had accessory pathways. The group with accessory pathways was younger than that of patients with re-entry of the atrioventricular node. Female sex and arterial hypertension were more frequently observed in patients with AVNRT, while male sex and the occurrence of AF-EPS predominated in cases with accessory pathways. Diabetes mellitus had a low prevalence in this sample and did not show differences between groups. There were no significant differences in body weight, heart rate, or atrial size when comparing both groups (Table 1). 
Table 2. P-wave parameters and atrial conduction times according to the occurrence or not of atrial fibrillation during the electrophysiological study

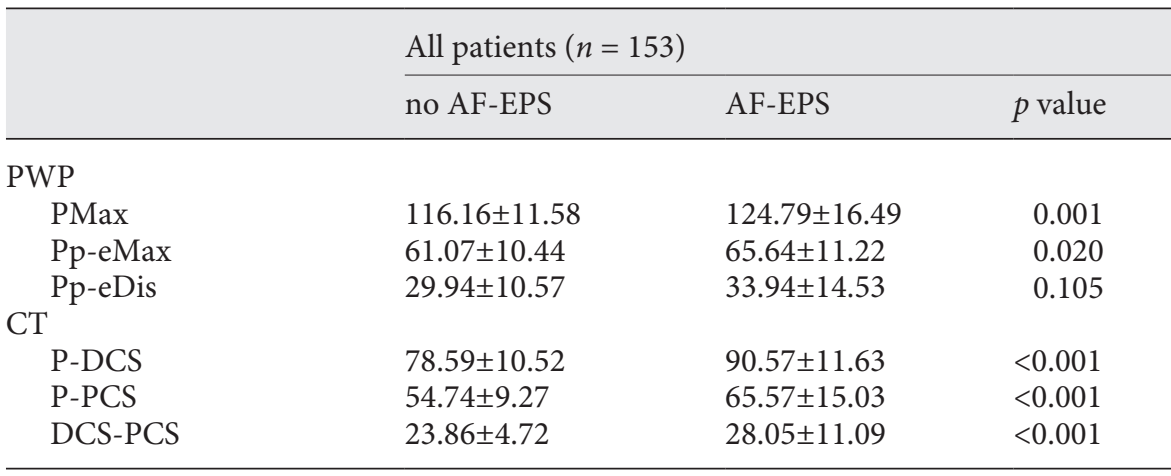

AF-EPS, atrial fibrillation in the electrophysiological study; CT, conduction times; DCS-PCS, left intra-atrial conduction time; P-DCS, P-distal coronary sinus interval; PMax, maximum P-wave duration; P-PCS, P-proximal coronary sinus interval; Pp-eDis, Ppeak-Pend interval dispersion; Pp-eMax, maximum Ppeak-Pend interval; PWP, P-wave parameters.
Table 3. Correlations between conduction times and $\mathrm{P}$-wave parameters according to the occurrence or not of atrial fibrillation during the electrophysiological study

\section{Basic Comparisons}

The 3 conduction times studied had a significantly longer duration in the group with AF-EPS compared to the group without AF-EPS. Of the P-wave parameters, only PMax and Pp-eMax were significantly higher in the AF-EPS group (Table 2).

\section{Correlations}

The $3 \mathrm{P}$-wave parameters were directly and significantly correlated with the P-DCS interval in cases with AF-EPS, and the same was observed in those without AFEPS. DCS-PCS correlated with all P-wave parameters.
The P-PCS interval was significantly correlated with PMax and Pp-eMax but only in the AF-EPS group. In general, the correlations were stronger in patients with AF-EPS (Table 3).

\section{Univariate and Multivariate Analysis}

In univariate logistic regression analysis, all conduction times, as well as the P-wave parameters, PMax and Pp-eMax, were significant predictors of AF-EPS (Table 4). In multivariate logistic regression analysis, it was observed that both PMax and Pp-eMax were independent predictors of AF-EPS, after adjustment for age, hyperten- 


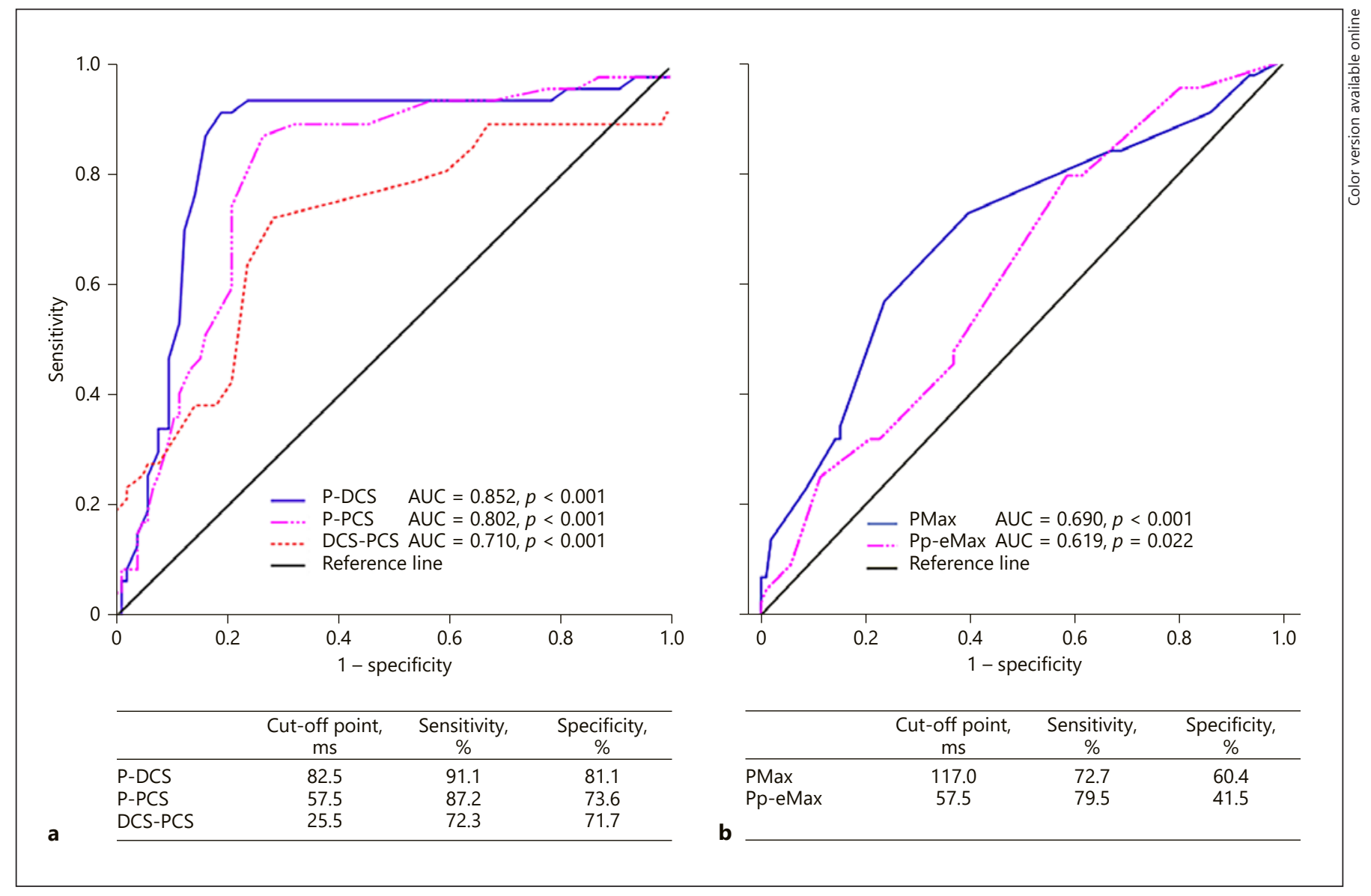

Fig. 1. ROC curves showing the relationship between sensitivity and specificity through all possible values of atrial conduction times and $\mathrm{P}$-wave parameters that define patients with atrial fibrillation in the electrophysiological study. a Analysis for atrial conduction times and $\mathbf{b}$ analysis for P-wave parameters. ROC, receiver operating characteristic; AUC, area under the ROC curve; DCS-PCS, left intra-atrial conduction time; P-DCS, P-distal coronary sinus interval; PMax, maximum P-wave duration; P-PCS, P-proximal coronary sinus interval; $\mathrm{Pp}$ eMax, maximum Ppeak-Pend interval.

Table 4. Results of the univariate and multivariate binary logistic regression analysis based on the prediction of atrial fibrillation during the electrophysiological study by atrial conduction times and P-wave parameters

\begin{tabular}{|c|c|c|c|c|c|c|}
\hline & \multicolumn{3}{|c|}{ Univariate analysis $(N=153)$} & \multicolumn{3}{|c|}{ Multivariate analysis* $(N=153)$} \\
\hline & OR & $p$ value & $95 \%$ CI & OR & $p$ value & $95 \% \mathrm{CI}$ \\
\hline DCS-PCS & 1.088 & 0.004 & $1.028-1.151$ & 1.092 & 0.006 & $1.025-1.163$ \\
\hline P-DCS & 1.101 & $<0.001$ & $1.059-1.146$ & 1.101 & $<0.001$ & $1.059-1.144$ \\
\hline P-PCS & 1.090 & $<0.001$ & $1.047-1.135$ & 1.097 & $<0.001$ & $1.053-1.144$ \\
\hline PMax & 1.048 & 0.001 & $1.020-1.078$ & 1.055 & 0.001 & $1.024-1.088$ \\
\hline Pp-eMax & 1.039 & 0.021 & $1.006-1.074$ & 1.046 & 0.012 & $1.013-1.079$ \\
\hline Pp-eDis & 1.028 & 0.063 & $0.998-1.058$ & 1.006 & 0.072 & $0.976-1.036$ \\
\hline
\end{tabular}

CI, confidence interval; DCS-PCS, intra-left atrial conduction time; OR, odds ratio; P-DCS, P-distal coronary sinus interval; PMax, maximum P-wave duration; P-PCS, $\mathrm{P}$-proximal coronary sinus interval; $\mathrm{Pp}$-eDis, $\mathrm{P}$ peak- $\mathrm{P}$ end interval dispersion; $\mathrm{Pp}$-eMax, maximum $\mathrm{P}$ peak-P end interval duration. * Adjusted for age, hypertension, arrhythmic diagnosis, and left and right atrial size. 
sion, arrhythmic diagnosis, and left and right atrial size (Table 4). P-PCS, P-DCS, and DCS-PCS were also independent predictors, after adjustment for the same variables (Table 4).

\section{Discriminatory Ability of Atrial Conduction Times}

and P-Wave Parameters for AF-EPS - ROC Curves

In general, the area under the ROC curve of the electrophysiological parameters as a function of predicting AF-EPS was significant for the 3 atrial conduction times (DCS-PCS, P-DCS, and P-PCS) (Fig. 1a). The best sensitivity and specificity values were obtained for P-DCS and P-PCS (bottom, Fig. 1a). PMax and Pp-eMax showed significant areas under the curve (Fig. 1b). Pp-eMax was a more sensitive marker of AF-EPS than PMax but much less specific (bottom, Fig. 1b).

\section{Discussion}

Our finding that patients with AF-EPS have longer atrial conduction times can be explained by the role played by fibrosis, anisotropy, and electrical remodeling in conduction disturbances. The patients studied presented arrhythmic substrates that could also influence the results. Deniz et al. [13] studied 86 cases with supraventricular arrhythmias by EPS and in whom AF was induced by rapid stimulation; they found that patients with induced $\mathrm{AF}$ had higher values in interatrial conduction time $(67 \pm 15 \mathrm{~ms}$ vs. $55 \pm 14 \mathrm{~ms} ; p<0.001)$ and DCS-PCS $(27 \pm 10 \mathrm{~ms}$ vs. $19 \pm 8 \mathrm{~ms} ; p<0.001)$. In an electroanatomic mapping study that compared the right and left atrial conduction velocity in patients with AF and AVNRT (control group), a significant decrease in this was observed in the group with AF, both in the right and left atrium, with lower values in the latter [14]. Even in patients with persistent $\mathrm{AF}$, the electromechanical delay is greater than in patients with paroxysmal $\mathrm{AF}$, which can be explained by the more advanced degree of remodeling in the former, highlighting the role that this plays in AF [15].

The finding of direct and significant correlations between the parameters of the second half of the P-wave and the electrophysiological parameters of atrial conduction is a novel observation. Our finding suggests that measurements of the second half of the P-wave from the surface electrocardiogram could prove to be of value for predicting clinical AF. Correlation was best for P-DCS and DCSPCS, supporting the hypothesis of our research, namely, that these variables better characterize left atrial conduc-

Predictors of Atrial Fibrillation during

Electrophysiological Studies tion. Similar correlations have been reported for PMax and $\mathrm{P}$-wave dispersion in other studies [16]. In a casecontrol study, Liu et al. [6] found that PMax had a longer duration in subjects with paroxysmal AF than in those without. PMax also correlated with conduction alterations during EPS. Ermis et al. [7] found that PMax significantly correlated with intra- and interatrial conduction times in prehypertensive patients, also using the same measurement method. In nondipper hypertensive patients, longer atrial electromechanical delay times were seen together with higher values of PMax, $\mathrm{P}$-wave dispersion, and $\mathrm{P}$-wave terminal force in lead $\mathrm{V} 1$, in addition to lower values in the parameters of left atrial strain [11].

Several P-wave parameters on the surface electrocardiogram, particularly "interatrial block," are good predictors of AF $[17,18]$. In elderly patients enrolled in the PREDICTOR study, interatrial block strongly predicted AF indicating more than triple the risk [19]. In a meta-analysis that included 18,204 patients, Tse et al. [20] showed that interatrial blocks are significant predictors of both new-onset AF and AF recurrence. Recently, the MVP (morphology-voltage-P-wave duration) score was published, for the prediction of new-onset AF [21]. Using the MVP risk score, the patients were classified as low, intermediate, and high risk. In these last 2 groups, the appearance of AF was more likely (odds ratio: 2.4; 95\% CI: $1.3-$ 4.4; $p=0.006$, and odds ratio: $2.1 ; 95 \%$ CI: $1.4-3.27 ; p=$ 0.009 , respectively). Other important $\mathrm{P}$-wave parameters in the prediction of $\mathrm{AF}$ are $\mathrm{P}$-wave axis, $\mathrm{P}$-wave duration (maximum, minimum, and mean), $\mathrm{P}$-wave area, $\mathrm{P}$-wave terminal force in lead V1, signal-averaged $\mathrm{P}$-wave duration, and $\mathrm{P}$-wave dispersion [22].

$\mathrm{P}$-wave duration is also a recognized predictor of $\mathrm{AF}$. The fibrotic and fatty changes in the atria that disrupt the electrical flow and result in prolonged $\mathrm{P}$-wave duration also facilitate AF [23]. The cutoff value reported in this investigation for PMax is close to $120 \mathrm{~ms}$, which is the cutoff point used to diagnose partial interatrial block. The novelty would be its predictive capacity when there is still no clinical evidence of AF, since AF-EPS should be interpreted as an indicator of increased electrical vulnerability. $\mathrm{P}$-wave duration is a reflection of the time required for right and left atrial depolarization [22]. This became evident from its good correlation with P-DCS, which reflects total atrial conduction time. There is evidence that measurement of these intervals can reveal atrial conduction defects even in the absence of $\mathrm{P}$-wave prolongation [24], making them very sensitive predictors. Therefore, the result of a lower discriminative capacity of the P-wave parameters with respect to conduction times is expected. 
The $\mathrm{P}$-wave is produced by the interaction of electrophysiological, vectorial, geometric, and body surface factors that must be taken into account in its interpretation.

The higher prevalence of AVNRT in women has been previously reported, while male gender, younger age, and occurrence of AF are more prevalent in patients with accessory pathways $[25,26]$. To the best of our knowledge, except for the study by Morris [27], no other studies have evaluated the parameters of the second half of the P-wave and their value in predicting AF. Of the 2 new parameters initially proposed (Pp-eMax and $\mathrm{Pp}$-eDis), only $\mathrm{Pp}$-eMax showed discriminative capacity to identify cases with AF, slightly surpassing the sensitivity of PMax, albeit with less specificity. The good correlation observed between DCSPCS and Pp-eMax indicates that this P-wave parameter characterizes the left intra-atrial conduction time well. Other predictors such as interatrial blocks have shown a more robust predictive capacity than those reported here. However, they have always been studied in patients who clinically manifest AF; in addition, the advanced form of these blocks that has been most favored by research is not frequently observed.

Limitations of our study include the noninclusion of cases with a previous history of AF and the small sample size. In addition, most patients had AVNRT which can limit the generalization of our findings. In the present study, the analyzed P-wave parameters were able to predict AF-EPS, which undoubtedly suggests that they could represent powerful markers of increased risk for clinical AF. Our greatest contribution lies in relating the findings in the $\mathrm{P}$-wave parameters with those that invasively assess atrial conduction and the proposal of a new electrocardiographic predictor.

\section{Conclusions}

Left atrial conduction times directly and significantly correlate with PMax and almost all of the parameters of the second half of the P-wave. PMax and Pp-eMax (new parameter) were good predictors of AF-EPS, although the conduction times did more robustly.

\section{Statement of Ethics}

All patients included in the study gave their informed consent to carry out the electrophysiological study and radiofrequency endocavity ablation. The local ethics committee approved this study.

\section{Conflict of Interest Statement}

The authors have no conflicts of interest to declare.

\section{Funding Sources}

This research received no grant from any funding agency in the public, commercial, or not-for-profit sectors.

\section{Author Contributions}

All authors were involved in the planning and execution of this study. R. Carmona Puerta, E. Lorenzo Martinez, G. Padrón Peña, J.M. Cruz Elizundia, F. Rodríguez González, and E. Chávez González: collection of clinical data. R. Carmona Puerta and E. Lorenzo Martinez: analysis of raw data and drafting of the manuscript. R. Carmona Puerta, M. Rabassa López-Calleja, Y. Castro Torres, and L.A. García Vázquez: review of the manuscript.

\section{References}

1 Chen Q, Gasperetti A, Della Rocca DG, Mohanty S, Gedikli O, Trivedi C, et al. The value of baseline and arrhythmic ECG in the interpretation of arrhythmic mechanisms. Card Electrophysiol Clin. 2019;11:219-38.

2 Carmona Puerta R, Lorenzo Martínez E. P wave dispersion over two decades after its discovery. What the cardiologist should know. CardioClinics. 2021;56(1):49-57.

3 Maheshwari A, Norby FL, Roetker NS, Soliman EZ, Koene RJ, Rooney MR, et al. Refining prediction of atrial fibrillation-related stroke using the P2-CHA2DS2-VASc score: ARIC and MESA. Circulation. 2019;139:18091.

4 Magnani JW, Zhu L, Lopez F, Pencina MJ, Agarwal SK, Soliman EZ, et al. P-wave indices and atrial fibrillation: cross-cohort assess- ments from the Framingham heart study (FHS) and atherosclerosis risk in communities (ARIC) study. Am Heart J. 2015;169:53e1.

5 Platonov PG. Atrial conduction and atrial fibrillation: what can we learn from surface ECG? Cardiol J. 2008;15:402-7.

6 Liu Z, Hayano M, Hirata T, Quin Y, Tsukahara $\mathrm{K}$, Ishimatsu $\mathrm{T}$, et al. Abnormalities of electrocardiographic $\mathrm{P}$ wave morphology and the relationship to electrophysiological parameters of the atrium in patients with idiopathic paroxysmal atrial fibrillation. J Cardiol. 1998;32:189-96.

7 Ermis N, Acikgoz N, Yasar E, Tasolar H, Yagmur J, Cansel M, et al. Evaluation of atrial conduction time by $\mathrm{P}$ wave dispersion and tissue Doppler echocardiography in prehyper- tensive patients. Turk Kardiyol Dern Ars. 2010;38:525-30.

8 Emiroglu MY, Bulut M, Sahin M, Acar G, Akcakoyun M, Kargin R, et al. Assessment of atrial conduction time in patients with essential hypertension. J Electrocardiol. 2011;44: 251-6.

9 Akturk E, Yağmur J, Açıkgöz N, Ermiş N, Cansel M, Karakus Y, et al. Assessment of atrial conduction time by tissue Doppler echocardiography and $\mathrm{P}$-wave dispersion in smokers. J Interv Card Electrophysiol. 2012; 34:247-53.

10 Zehir R, Karabay CY, Kocabay G, Kalayci A, Kaymaz O, Aykan AC, et al. Assessment of atrial conduction time in patients with polycystic ovary syndrome. J Interv Card Electrophysiol. 2014;41:137-43. 
11 Tosun V, Korucuk N, Kılınç AY, Uygun T, Altekin RE, Güntekin Ü, et al. Evaluation of atrial electromechanical functions in dipper and nondipper hypertension patients using left atrial strain $\mathrm{P}$-wave dispersion and $\mathrm{P}$ terminal force. Echocardiography. 2018;35: 1318-25.

12 Lang RM, Badano LP, Mor-Avi V, Afilalo J, Armstrong A, Ernande L, et al. Recommendations for cardiac chamber quantification by echocardiography in adults: an update from the American Society of Echocardiography and the European association of cardiovascular imaging. Eur Heart J Cardiovasc Imaging. 2015;16:233-70.

13 Deniz A, Sahin DY, Kanadasi M, Demir M, Berk IG, Akkus O, et al. Conduction characteristics in atrial fibrillation. Predictive value of tissue Doppler echocardiography. Herz. 2014;39:137-41.

14 Zheng Y, Xia Y, Carlson J, Kongstad O, Yuan S. Atrial average conduction velocity in patients with and without paroxysmal atrial fibrillation. Clin Physiol Funct Imaging. 2017; 37:596-601.

15 Chen X, Liu H, Zhang Y, Wang C, Xu D, Yang $\mathrm{B}$, et al. Atrial electromechanical delay assessment in early phase after catheter ablation for patients with atrial fibrillation. Pacing Clin Electrophysiol. 2019;42:327-32.
16 Carmona Puerta R, Chávez González E, Rabassa López-Calleja MA, Lorenzo Martínez E, Cruz Elizundia JM, Padrón Peña G, et al. Atrial conduction explains the occurrence of the $\mathrm{P}$-wave dispersion phenomenon, but weakly. J Arrhythmia. 2020;36(6):1083-91.

17 Bayés de Luna A, Martínez-Sellés M, BayésGenís A, Elosua R, Baranchuk A. Sindrome de Bayes. Lo que todo clinico debe conocer. Rev Esp Cardiol. 2020;73:758-62.

18 Vicent L, Fernández-Cordón C, NombelaFranco L, Escobar-Robledo LA, Ayesta A, Ariza Solé A, et al. Baseline ECG and prognosis after transcatheter aortic valve implantation: the role of interatrial block. J Am Heart Assoc. 2020;9(22):e017624.

19 Boccanelli A, Mureddu GF, Cesaroni G, Prati F, Rangoni F, Agabiti N, et al. Predictive value of interatrial block for atrial fibrillation in elderly subjects enrolled in the PREDICTOR study. J Electrocardiol. 2019;54:22-7.

20 Tse G, Wong CW, Gong M, Wong WT, Bazoukis $\mathrm{G}$, Wong $\mathrm{SH}$, et al. Predictive value of inter-atrial block for new onset or recurrent atrial fibrillation: a systematic review and meta-analysis. Int J Cardiol. 2018;250:152-6.

21 Alexander B, Milden J, Hazim B, Haseeb S, Bayes-Genis A, Elosua R, et al. New electrocardiographic score for the prediction of atrial fibrillation: the MVP ECG risk score
(morphology-voltage-P-wave duration). Ann Noninvasive Electrocardiol. 2019;24: e12669.

22 Chen LY, Soliman EZ. P wave indices-advancing our understanding of atrial fibrillation-related cardiovascular outcomes. Front Cardiovasc Med. 2019;6:53.

23 Hari KJ, Nguyen TP, Soliman EZ. Relationship between P-wave duration and the risk of atrial fibrillation. Expert Rev Cardiovasc Ther. 2018;16:837-43.

24 Weijs B, De Vos CB, Limantoro I, Cheriex EC, Tieleman RG, Crijns HJ. The presence of an atrial electromechanical delay in idiopathic atrial fibrillation as determined by tissue Doppler imaging. Int J Cardiol. 2012;156: 121-2.

25 Issa ZF, Miller JM, Zipes DP. Clinical arrhythmology and electrophysiology: A companion to Braunwald's heart disease. 3rd ed. China: Elsevier; 2019. p. 1067.

26 Chugh A, Morady F. Preexcitation, atrioventricular reentry, and variants. In: Zipes D, Jalife J, editors. Cardiac electrophysiology: from cell to bed side. 7th ed. Philadelphia: Elsevier Saunders; 2018. p. 736-45.

27 Morris JJ, Estes EH, Whalen RE, Thompson HK, Mcintosh HD. P-wave analysis in valvular heart disease. Circulation. 1964;29:24252. 